\title{
Calorimetric properties of mesoscopic superconducting disks, rings, and cylinders
}

\author{
Ben Xu, M. V. Milošević, and F. M. Peeters* \\ Departement Fysica, Universiteit Antwerpen, Groenenborgerlaan 171, B-2020 Antwerpen, Belgium \\ (Received 8 October 2009; revised manuscript received 6 January 2010; published 1 February 2010)
}

\begin{abstract}
The thermal signatures of superconductivity in mesoscopic disks, rings and cylinders are calculated within the Ginzburg-Landau theory. In an applied perpendicular magnetic field $H$ the heat capacity of mesoscopic samples shows a strong dependence on the realized vortex state; discontinuities are found at the critical field for different vorticities, as well as at the superconducting-to-normal state transition. The same applies to the intermediate state of type-I superconductors. Even the subtle changes in the fluxoid distribution inside the sample leave clear signatures on heat capacity, which is particularly useful for fully three-dimensional samples whose interior is often inaccessible by magnetometry. The heat-capacity jump $\Delta C(H)$ at the critical temperature exhibits quasiperiodic modulations as a function of magnetic field. In mesoscopic superconducting rings, these oscillations provide calorimetric verification of the Little-Parks effect.
\end{abstract}

DOI: 10.1103/PhysRevB.81.064501

PACS number(s): 74.25.Bt, 74.25.Uv, 75.40.Cx, 74.78.Na

\section{INTRODUCTION}

Submicron superconducting devices are envisaged as a base for futuristic electronics, due to their low resistance and enhanced critical parameters compared to bulk materials. ${ }^{1,2}$ Mesoscopic superconducting devices already found a number of exotic applications, such as in astronomy as the microbolometer of time-resolved terahertz spectroscopy ${ }^{3}$ or as dark matter detectors. ${ }^{4}$ In latter systems, the intrinsic thermodynamic properties are of crucial importance, related to the energy needed for the heating of the system or to the heat released when the system changes its state. Yet, the theoretical understanding of such calorimetric properties of mesoscopic superconducting samples is in its infancy as compared to the extensively studied electromagnetic and transport properties of such samples.

In bulk superconducting materials, the specific heat shows sizeable jumps at the critical field for the superconducting/ normal $(\mathrm{S} / \mathrm{N})$ state transition, which was one of the most important proofs for the existence of superconducting condensation. ${ }^{5}$ In type-II superconducting materials, the experimental and analytical analysis of the specific heat were done in Refs. 6 and 7 as a function of applied magnetic field $H$. They found that the amplitude of the jump in the heat capacity at the $\mathrm{S} / \mathrm{N}$ transition decreases as the applied magnetic field is increased (simultaneously, the critical temperature decreases). Another important aspect of this work is that, when the specimens are heated in a constant magnetic field, the heat capacity exhibits a very large peak at low temperature followed by a discontinuous drop in heat capacity at a higher temperature. The latter is connected with the secondorder $\mathrm{S} / \mathrm{N}$ transition at $H=H_{c 2}$. Similar phenomenon was also found in disordered superfluid ${ }^{3} \mathrm{He},{ }^{8}$ as the specific heat of superfluid ${ }^{3} \mathrm{He}$, disordered by a silica aerogel, is found to have a sharp discontinuity marking the thermodynamic transition to superfluidity (with lower amplitude and at a reduced temperature from that of bulk ${ }^{3} \mathrm{He}$ ). The formerly described peak however is associated with the entrance of the magnetic flux into the specimen. 6 ,9

Calorimetric investigation of mesoscopic systems brings an innovative and complementary point of view to condensed-matter studies. ${ }^{10-12}$ Recently, it has become possible to study the heat capacity of submicron-sized superconducting samples due to the large progress in attoJoule calorimetry. ${ }^{11,13}$ The first heat-capacity measurements of the phase transitions between vortex states in mesoscopic singly connected superconductors were recently performed by Bourgeois et al. ${ }^{11}$ and Ong et al. ${ }^{14}$ The experiments were carried out on large arrays of mesoscopic two-dimensional disks and rings, to maximize the output signal. Heat-capacity curves versus temperature or field were found to clearly indicate the number of vortices threading the sample, where each entrance or exit of a vortex caused a jump from one heat-capacity level to another. As for superconducting rings, oscillations in the size of the discontinuity of the heat capacity at the $\mathrm{S} / \mathrm{N}$ transition were found and the measured oscillations of $\Delta C_{p}$ with field showed a more pronounced amplitude than anticipated. The corresponding theoretical calculation and understanding is still lacking, and this is the primary objective of this work.

General theoretical description of the specific-heat behavior of the mixed state in superconductors was first discussed by de Gennes ${ }^{15}$ and Fetter. ${ }^{16}$ At low and intermediate fields, the difference of the specific heat between vortex states and the fully superconducting state is proportional to $-B T\left(\partial^{2} H_{c 1} / \partial T^{2}\right) / 4 \pi$, and also to a free-energy functional $\mathcal{F}[H]$, which is complicated and must be calculated numerically (especially for a mesoscopic sample containing a dense distribution of vortices). In his analysis, de Gennes also described the specific-heat change between two different vortex states, which is proportional to the obtained difference in $\left(\partial B_{i} / \partial H\right),{ }^{15}$ leading to the prediction of specific-heat jumps at magnetic fields for which a vortex enters or leaves the sample. Other attempts to describe calorimetric properties of superconductors have used the BCS theory or the Eilenberg equations. ${ }^{17}$ However, the applicability of latter approaches is limited to infinite periodic samples, due to the computational difficulties related to the mesoscopic boundary and the current-field coupling effects. Therefore, it is more convenient to study the thermal properties of these systems in the framework of a mean-field Ginzburg-Landau (GL) theory. For example, Bray $^{18}$ simulated the behavior of the specific heat in bulk and thin-film superconducting samples in a uni- 
form magnetic field, by the use of the "free fluctuation theory" and screening theory. Fink ${ }^{19}$ and Tinkham ${ }^{5}$ described the heat-capacity behavior in the case of a hollow superconducting cylinder and its relation to fluxoid quantization. Zhang ${ }^{20}$ extended these approaches to mesoscopic superconducting rings for susceptibility calculations and Deo et al. ${ }^{12}$ considered the heat capacity of superconducting disks, but restricted their analysis to the linearized Ginzburg-Landau theory.

There is therefore still a need for an approach that includes the influence of the quantized fluxoid states on the specific heat of mesoscopic samples, including the demagnetization effects, for mesoscopic samples of arbitrary geometry and size, plus for an arbitrary direction of applied magnetic field. This is an exact purpose of this paper; we present our state-of-the-art theoretical calculation, based on the full, nonlinear GL theory. This theory has been extensively used in the past to describe the electromagnetic properties of twodimensional superconductors, ${ }^{21}$ where now we carefully incorporate and study the thermal relationships of different factors, the kinetic energy of the condensate and the magnetic response of the sample. The present numerical approach allows us to describe not only two-dimensional (2D) mesoscopic superconducting systems but also mesoscopic threedimensional 3D samples, where the configuration of vortices is even more intricate due to the complex influence of the shape of the boundary and also the direction of the applied magnetic field. As we will show, in such cases, the heat capacity can be a useful tool to discriminate the delicate changes in vortex configurations.

We start from the specific-heat calculations for twodimensional mesoscopic superconducting disks and rings, where the relevant parameters are mostly taken from the experiments of Bourgeois and co-workers. ${ }^{10,14}$ The studied samples and theoretical formulation of the problem are presented in Sec. II. The calorimetric calculations for the disk geometry are presented in Sec. III. The corresponding thermal signatures of superconducting rings are shown in Sec. IV. Section V is devoted to 3D calorimetric response of superconducting cylinders, and our results are summarized in Sec. VI

\section{THEORETICAL APPROACH}

We consider mesoscopic superconducting disks and rings made of type-I material (Al), which are immersed in an insulator media and exposed to a perpendicular uniform magnetic field $H$. To be able to calculate the specific heat, we explicitly include the temperature dependence in the GL formalism for the description of the superconducting system. The superconducting-normal-state Gibbs free-energy difference in the GL approach can be expressed as

$$
\begin{aligned}
\mathcal{G}= & \mathcal{G}_{S H}-\mathcal{G}_{N H} \\
= & \int\left\{\alpha|\Psi|^{2}+\beta \frac{|\Psi|^{4}}{2}+\frac{1}{2 m^{*}}\left|\left(\frac{\hbar \nabla}{i}-\frac{e^{*} \vec{A}}{c}\right) \Psi\right|^{2}\right. \\
& \left.+\kappa^{2} \frac{(\vec{h}-\vec{H})^{2}}{8 \pi}\right\} d V,
\end{aligned}
$$

where the $\mathcal{G}_{S H}$ and $\mathcal{G}_{N H}$ correspond to the free energy of the sample in the superconducting and the normal state, respectively, at applied magnetic field $\vec{H}$ ( $\vec{h}$ denotes the resulting, total local field in and around the superconductor). ${ }^{5}$ The GL parameter $\kappa$ equals $\lambda / \xi$, where $\lambda$ is the penetration depth. Minimization of Eq. (1) leads to two coupled GL equations, the first being

$$
\left(\frac{\nabla}{i}-\vec{A}\right)^{2} \Psi+|\Psi|^{2} \Psi-(1-T) \Psi=0 .
$$

The derivation of the GL equations assumes the London gauge $\operatorname{div} \vec{A}=0$ for the vector potential $\vec{A}$. All variables in Eq. (2) are dimensionless: the distance is measured in units of the coherence length $\xi(T=0)$, the vector potential $\vec{A}$ in $c \hbar / 2 e \xi(0)$ [magnetic field in $H_{c 2}(0)=c \hbar / 2 e \xi(0)^{2}$ $\left.=\kappa \sqrt{2} H_{c}(0)\right]$, and the order parameter $\Psi$ is scaled to its value in the absence of the magnetic field.

However, the first GL equation is only sufficient for the description of extreme type-II samples or for an extremely thin superconducting film where screening effects can be neglected. Otherwise the demagnetization effects must be taken into account, and the second GL equation is needed,

$$
-\kappa^{2} \Delta \vec{A}=\frac{1}{2 i}\left(\Psi^{*} \vec{\nabla} \Psi-\Psi \vec{\nabla} \Psi^{*}\right)-|\Psi|^{2} \vec{A} .
$$

The right side of Eq. (3) is the density of the superconducting current $\vec{j}$ induced by the sample in response to the applied field. Note that for the quasi-2D system, i.e. small but non-negligible thickness $d$ of the sample, the variations of the magnetic field and order parameter in $z$ direction can be neglected, and Eq. (3) may be averaged over the sample thickness $d$. In that case, term $\kappa^{2}$ in Eq. (3) becomes the effective GL parameter $\kappa_{e}=\kappa^{2} \xi / d$.

Here we solve the GL equations self-consistently in a finite-difference scheme. ${ }^{22,23}$ We use the Neumann boundary conditions $\left.(-i \vec{\nabla}-\vec{A}) \Psi\right|_{\text {boundary }}=0$ for the order parameter on all sample surfaces, including the inner boundaries (if any). The convergent solution of Eqs. (2) and (3) determines the superconducting state corresponding to the local energy minimum. In search for all stable states for given conditions (thus the lowest energy ground state, and the higher energy metastable states), we repeat the calculation using different initial conditions. For a realistic comparison with a fieldcooled experiment, we initiate the calculation from randomly generated and very weak superconducting order parameter, while in a zero-field-cooled situation, we start from $|\Psi| \approx 1$ in the whole sample. Once a solution is found, we are able to calculate the specific heat of the superconducting state based on the relation

$$
C_{p}(H, T)=C(H, T)-C_{N}(H, T)=-T \frac{\partial^{2} \mathcal{G}}{\partial T^{2}},
$$

where $C$ denotes the total heat capacity of the sample, and $C_{N}$ is the heat capacity of the sample in the normal state [all in units of $\left.C_{0}=H_{c}^{2}(0) V /\left(8 \pi T_{c}\right)\right]$. In the calculations, we start from the equilibrium states and oscillate the temperature of the system with amplitude $10^{-4} T_{c 0}\left(T_{c}\right.$ at $\left.H=0\right)$, and then 


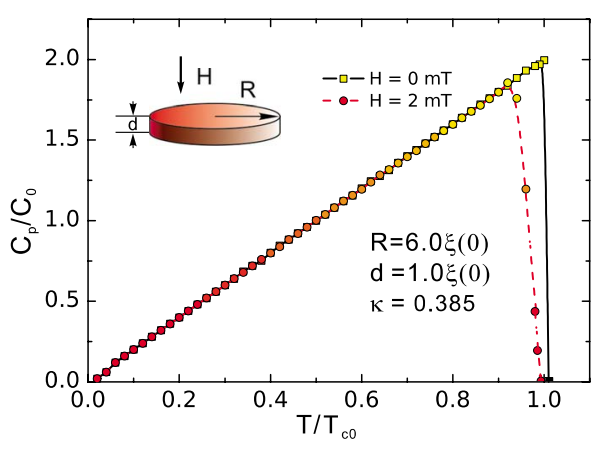

FIG. 1. (Color online) Heat capacity of a superconducting aluminum disk as a function of temperature in the absence and in presence of the magnetic field $H$. The calculation is done in the zero-field-cooled regime. Inset depicts the sample.

perform the second derivation numerically to obtain the specific heat for given parameters.

It should be mentioned here that solving Eq. (3) directly results in a finite magnetization of the sample. Instead of the thermodynamic expression for magnetization $M=\partial \mathcal{G} / \partial H$, we define in accordance with experimental reality, i.e., as the quantity of expelled magnetic field from the sample in applied field $H$,

$$
\vec{M}=\frac{\langle\vec{h}\rangle-\vec{H}}{4 \pi},
$$

where $\vec{h}=\operatorname{rot} \vec{A}$ is the local magnetic field in the sample, and \langle\rangle denotes averaging over the area of interest (e.g., a surface area of a magnetic detector at a given location with respect to the sample).

\section{HEAT CAPACITY OF MESOSCOPIC SUPERCONDUCTING DISKS}

In what follows, the sample is an aluminum disk of radius $R=6 \xi(0)$ and thickness $d=\xi(0)$ (see inset of Fig. 1). This corresponds to the experimental sample of Ong et al. ${ }^{14}$ where $\xi(0)$ was roughly estimated to $182 \mathrm{~nm}$ and $\lambda(0)$ to 70 $\mathrm{nm}$. This results in the GL parameter $\kappa \approx 0.385$, which is firmly in the type-I regime. Often in experiments $\mathrm{Al}$ samples are thinner than $\lambda(0),{ }^{24,25}$ which makes them effectively type-II $\left(\kappa_{e}>1 / \sqrt{2}\right)$, and conventional mesoscopic behavior of the superconducting state and vortex matter is found. In earlier works, ${ }^{21,26,27}$ the vortex entry and distinction between giant and multivortex states was thoroughly discussed. Surprisingly, Ong et al. ${ }^{14}$ also discussed their findings from the standpoint of a type-II sample. While modeling the latter experiment, we correct this somewhat, as explained further.

Conventionally, the behavior of the superconducting state in mesoscopic type-II superconductors as a function of applied magnetic field is discussed with respect to the upper critical field $H_{c 2}$. In the present case, we scale the field to the thermodynamic critical field $H_{c}$, which in our sample equals $H_{c}(0)=\Phi_{0} / 2 \sqrt{2} \pi \xi(0) \lambda(0) \approx 18.34 \mathrm{mT}$, with $H_{c}(T)$ $=H_{c}(0)\left(1-T / T_{c}\right)$. Since penetration depth $\lambda(0)$ is comparable to the thickness $d$ and increases with temperature, a homogenous distribution of magnetic field across the sample thickness can be assumed. Nevertheless, we use the threedimensional treatment of the two coupled Ginzburg-Landau equations, taking thus fully into account the 3D demagnetization effects. We are interested in two calorimetric sets of data-one for the heat capacity versus magnetic field under fixed temperature, and the other for the case of fixed magnetic field, and the variation of the heat capacity versus temperature.

In Fig. 1 the $C_{p}\left[=C_{\text {total }}-C_{N}\right]$ curve for the forementioned superconducting disk is presented as a function of temperature (scaled to $T_{c 0}$ ). When present, the field was always applied after the sample had been cooled down to zero temperature, i.e., we work in the zero-field-cooled regime. We subsequently increase the temperature gradually. We monitor the heat capacity of the Meissner state, although at higher temperatures vortices may enter the system in the ground state. However, due to the finite Bean-Livingston barrier and the absence of fluctuations, ${ }^{28}$ fluxoids are generally unable to enter the sample during the temperature sweep. In absence of applied field, the $L=0$ state shows a discontinuity at $T_{c}$, analogously to the behavior of bulk superconductors (see Fig. 1). The critical temperature is often determined by the temperature at half-height of the $C_{p}$ jump, and the size $\Delta C(H)$ of the heat-capacity discontinuity can be extracted at the $\mathrm{S} / \mathrm{N}$ transition.

In their attoJoule measurements, Ong et al. ${ }^{14}$ showed the possibility of modulating the heat capacity of mesoscopic disks by a magnetic field and investigated the response of the different vortex states. Namely, the heat capacity is directly linked to the flux distribution inside the sample and the resulting vorticity. Since the corresponding theoretical calculations are missing up to date, we perform here the numerical "experiment:" we fix the temperature in the absence of applied field (to $0.5 T / T_{c 0}$ ), and then sweep up the magnetic field continuously toward the destruction of superconductivity, while recording all found vortex states along the way. Subsequently, we decrease the magnetic field down to zero, again in search for stable vortex states. For each recorded equilibrium state, we then vary the temperature with $\Delta T$ $=10^{-4} T_{c 0}$ from the original one, in order to calculate the variation of the free energy and the heat capacity according to Eq. (4). The temperature step is kept small to ensure the precision of the calculation, but also to avoid any change in the original vortex state.

At this point, we should clarify that found vortex states in the experiment are not the signature of type-II superconductivity. To show this in comparison with experimental data, we scale the applied magnetic field value to $H_{c}(T)$ and specific heat to $C_{0} ; C_{0}$ equals $H_{c}^{2}(0) V /\left(8 \pi T_{c 0}\right)(V$ is the sample volume), which amounts to $4.5 \times 10^{-5} \mathrm{pJ} / \mathrm{K}$ for a single disk, but equals $18.9 \mathrm{pJ} / \mathrm{K}$ for large arrays of the samples (number of disks $N=4.2 \times 10^{5}$ ) as was the case in the experiment.

The obtained heat capacity $C_{p}(H, T)$ versus magnetic field for fixed temperature is shown in Fig. 2(a). Starting from zero field, the disks remain in the Meissner state $(L=0)$ up to $H \approx 0.30 H_{c}(T)$ where $C_{p}$ abruptly jumps to a lower value. This is the calorimetric signature of the first-order phase transition $L=0 \rightarrow L=1$, where one vortex penetrates the disk. 


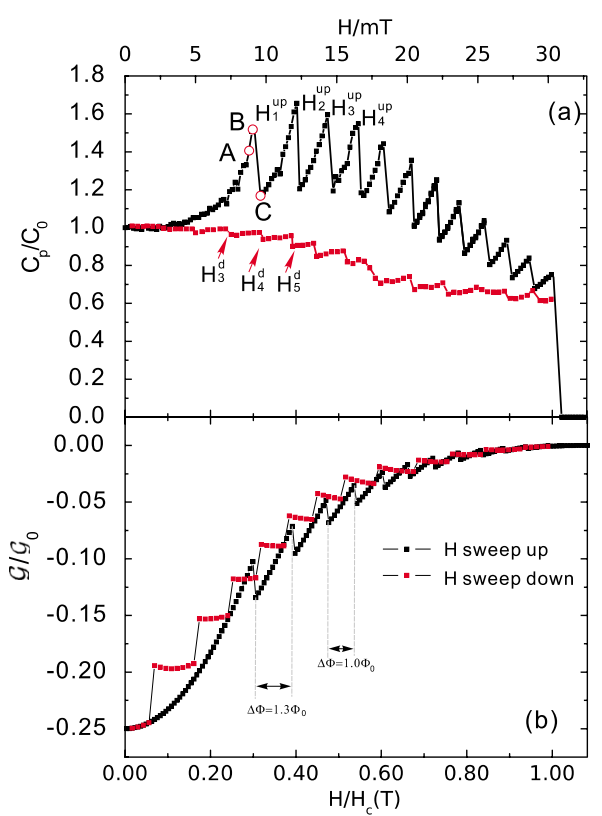

FIG. 2. (Color online) (a) Heat capacity $\mathrm{C}_{p}(H, T)$ is plotted as a function of the applied magnetic field at temperature $T=0.5 T_{c 0}$, for increasing and decreasing magnetic field. The data shows pronounced hysteretic behavior. $H_{L}^{u p}$ shows the penetration field of the $L-t h$ vortex in the system and $H_{L}^{d}$ is the vortex expulsion field (the transition to a $L-1$ state). (b) The free-energy curves versus the magnetic field for the magnetic field swept up and down. The unit of the free energy $\mathcal{G}_{0}$ is $H_{c}^{2}(0) / 8 \pi$.

As the field is increased further, we observe a cascade of successive phase transitions $L \rightarrow L+1$, until the superconducting/normal second-order transition is reached. Although this scenario perfectly resembles the type-II samples, the found vortex states actually represent the intermediate state of a type-I superconductor. The ratio between the thickness and the diameter of the sample is $1 / 12$, and using the calculation of the demagnetizing field of a largest inscribed ellipsoid from Ref. 29, we found that at applied field of $H \approx 0.30 H_{c}(T)$ the field at the sample edges equals $H_{c}(T)$ and flux penetrates our type-I sample (see also Fig. 7 in Ref. 29). Also in accordance with the conventional theory of type-I superconductivity, the superconducting state is finally destroyed at $H \approx H_{c}$.

Note that due to the mesoscopic size of our type-I sample, all found flux states are giant-vortex states. After the first penetration field, the stability field region for the following vortex state is approximately $0.09 H_{c}(T)$. Latter value provides for added flux of $\Delta \Phi \approx 1.3 \Phi_{0}$ through the system, and corresponds well to the experimental data (Fig. 4 in Ref. 14). As indicated in Fig. 2, the added flux decreases with further increasing vorticity to $1.0 \Phi_{0}$, showing that the flux quantization applies to the entire sample at high fields (at lower fields the flux is quantized inside the zero-current path, located between the giant vortex and the encircling Meissner currents). The total flux at the penetration field for the $L=1$ vortex state is much higher than for the successive vortex states, because of the superheating of the Meissner phase, and is readily observed even in type-II samples. ${ }^{21}$ Note the different definition of the heat capacity in our calculation compared to the experiment; in experiment, the superconducting part of the specific heat is shown after subtraction of the whole background from the measured signal, while in our calculation $C_{\text {total }}(T, H)-C_{N}(T, H)$ is used. As a result, we have a different starting value of the heat capacity of the Meissner phase in the absence of magnetic field, and a different background curvature while changing the magnetic field.

The behavior of the heat capacity of the superconducting disk in increasing magnetic field can be explained by considering the temperature dependence of the different terms in the free energy in the Ginzburg-Landau theory. For $H<H_{L}^{u p}$ (the penetration field of the $L-t h$ vortex in the system, where the $L-1 \rightarrow L$ transition takes place), we observe an increase in the specific heat with increasing $H$. In the microscopic scheme of the BCS theory, the increase of temperature excites more quasiparticles in the system, and the well-known temperature dependence of the energy gap $\Delta$ can be derived $\frac{\Delta(T)}{\Delta(0)} \approx 1.74\left(1-\frac{T}{T_{c}}\right)^{1 / 2}$, for temperatures close to $T_{c}$. In the Ginzburg-Landau theory, the temperature dependence of the order parameter resembles the one of the energy gap $\Delta$; i.e., the density of Cooper pairs ceases with temperature and decreases the heat capacity of the superconducting sample. This contribution enters the free energy through the term containing $|\psi|^{4}$. However, in mesoscopic samples, we find that the temperature dependence of the field-dependent term in the Ginzburg-Landau free energy is of crucial importance [in reduced form of the free energy, this term can be written as $\left(\vec{A}-\vec{A}_{0}\right) \cdot \vec{j}$, where $\vec{A}_{0}$ is the vector potential of the applied field, and $\vec{j}$ is the supercurrent, see Ref. 21].

Lets consider the above two different contributions of the free energy to the total heat capacity, specifically the $|\psi|^{4}$ term $\left(C_{\Psi}\right.$, related to the depletion of the condensate) and the other from the superconducting current contribution $\left(C_{J}\right.$, related to the kinetic energy of the condensate), which we show in Fig. 3. The total heat capacity shows a cascade of discontinuities for different vortices, but the major contribution of these jumps is different for low and high vorticity. For example, $C_{\Psi}$ contributes mostly to the plateau of the vortex states of $L=1$ and $L=2$, but the steep background of $C_{p}$ is due to $C_{J}$. Looking at $C_{J}$, the difference between the vortex states at their critical fields are present even for higher vorticity states, but those jumps are small compared to the oscillations of $C_{\Psi}$. In these calculations, while keeping $\xi(0)=182 \mathrm{~nm}$, we took the Ginzburg-Landau parameter $\kappa_{e}=1.18$, thus clearly type II.

The two contributions $C_{J}$ and $C_{p}$ to the specific heat can be investigated for different materials, i.e., with different Ginzburg-Landau parameter $\kappa^{33}$ To compare with Fig. 3, we now show the total heat capacity and its two components $C_{J}$ and $C_{p}$ for different vortex states when $\kappa_{e}=0.64$ (Fig. 4), thus just under the type-I/II transition. In both figures, one can find that the difference of $C_{\Psi}$ between adjacent vortex states is small for lower vorticity, while the amplitude of the oscillation is much higher for higher vorticity, especially those close to the $\mathrm{S} / \mathrm{N}$ transition point. It is these high vorticity oscillations that contribute most to the behavior of the total heat capacity at high magnetic field. $C_{J}$ shows a larger oscillation amplitude for smaller $\kappa$, i.e., more than a factor of 


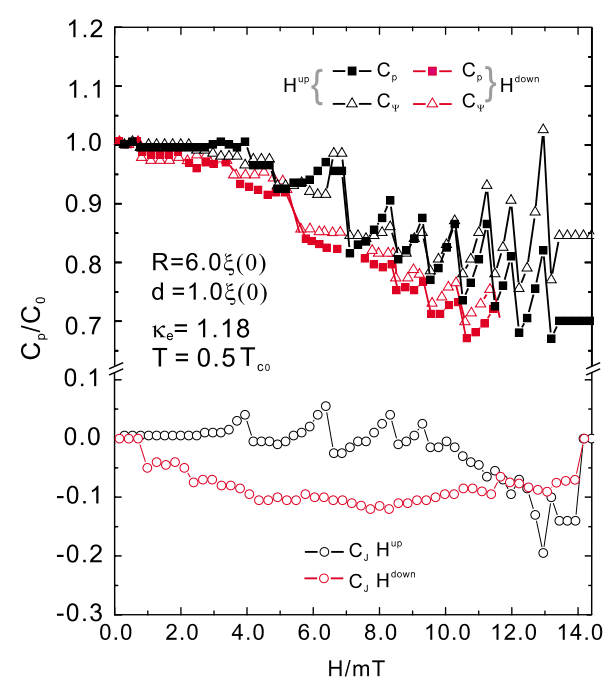

FIG. 3. (Color online) The heat capacity (filled squares) is plotted for a disk sample with Ginzburg-Landau parameter $\kappa_{e}=1.18$. The two components of the heat capacity $C_{\Psi}$ (open triangles) and $C_{J}$ (open dots) are shown. The calculations are done for sweeping up and down the magnetic field.

2 larger for $\kappa_{e}=0.64$ as compared to $\kappa_{e}=1.18$ because of the strong Meissner currents in the type-I sample. Also interesting are the vortex states (and values of the magnetic field) where the contribution $C_{J}$ drops from positive to negative. In Fig. 3, such switching happens several times, along the curves of the vortex states $L=1-3$, while in the case of $\kappa_{e}$ $=0.64$, it happens only for vorticity $L=1$, and remains negative afterwards. Therefore, type-I samples are more susceptible to heating at high fields than the type-II ones.

In general, we can conclude that the major contribution to the lower vorticity oscillations is due to the superconducting current, and at high vorticity due to the depletion of the condensate. The heat capacity decreases with increasing applied magnetic field, and the size of the decline is controlled by the Ginzburg-Landau parameter $\kappa$.

To obtain a more detailed understanding of $C_{J}$ for lower vorticity states, we plot the calculated distribution of the su-

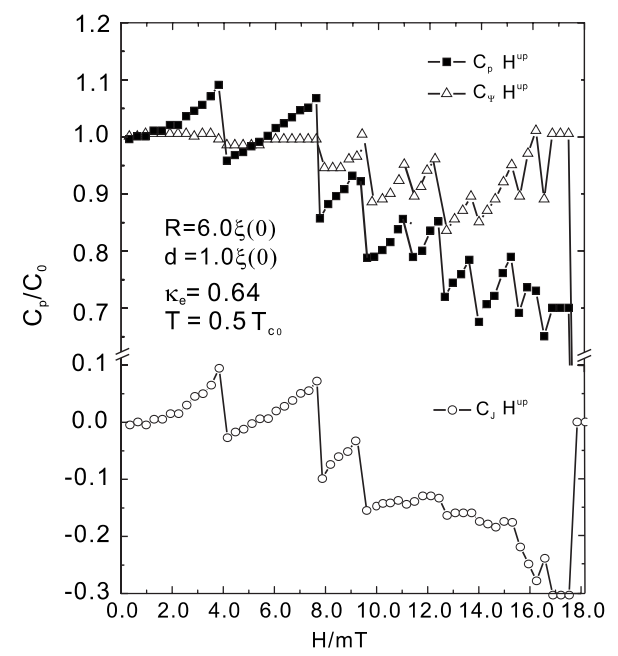

FIG. 4. The same as Fig. 3 but now for $\kappa_{e}=0.64$.

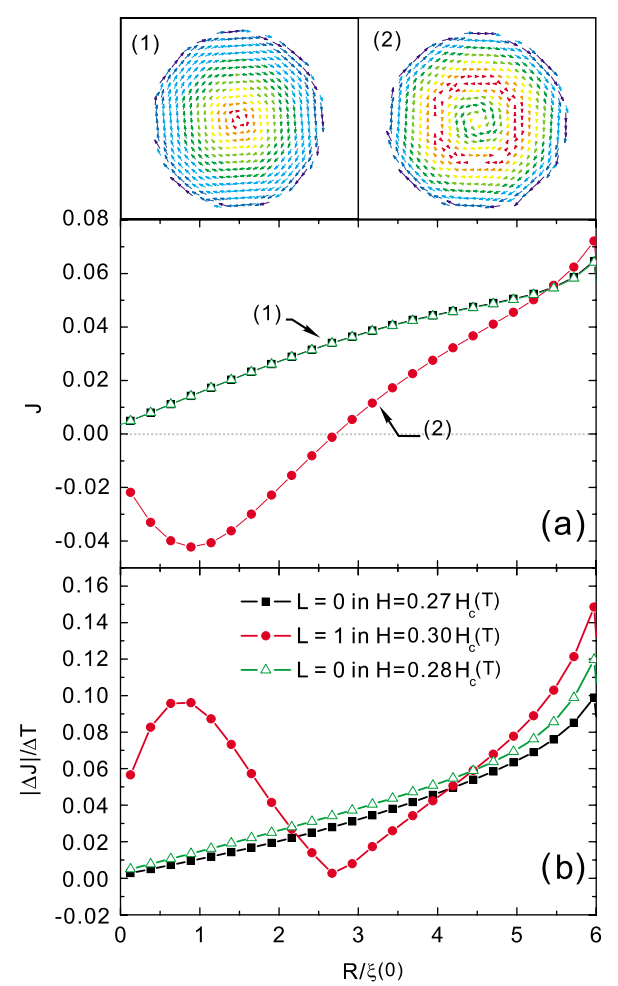

FIG. 5. (Color online) (a) The distribution of the azimuthal superconducting current in the radial direction inside the superconducting disk, for the same parameter as used in Fig. 2, for $H=0.27,0.28$, and $0.30 H_{c}(T)$. Insets (1-2) show the vector plots of the current for vorticity 0 and 1 , with magnitude indicated by color coding changing from red (minimum) to blue (maximum). (b) The variation of current shown in (a) with temperature $(\partial j / \partial T)$, for applied field $H=0.27 H_{c}(T)$ (system in the Meissner state, point A in Fig. 2), field $H=0.28 H_{c}(T)$ (close to $H_{1}^{u p}$, point B in Fig. 2), and field $H=0.30 H_{c}(T)$ (point $\mathrm{C}$ in Fig. 2, $L=1$ state).

percurrent in Fig. 5. As shown in the insets, the current has only an azimuthal component, and is cylindrically symmetric for the shown states. Three characteristic magnetic fields are chosen: (i) the field for which the system is in the Meissner state, where the superconducting current preserves a high density of superconducting order parameter; (ii) the field prior to the $L=0 \rightarrow 1$ transition, and (iii) at the $L=0 \rightarrow 1$ transition. When the magnetic field is increased for the system in the $L=0$ state, the density of the superconducting current increases in order to expel the applied magnetic field. The maximum of the current density is reached prior to the penetration field, upon which the current becomes bipolaranticlockwise inside the vortex core, and clockwise otherwise [see insets of Fig. 5(a)]. Figure 5(b) shows the variation of the superconducting current with temperature, calculated for a temperature increase by $\Delta T=0.005 T_{c 0}$. The variation of the current achieved for $L=1$ is much larger than that of the $L=0$ state, which reflects the observed difference in the heat capacity. Namely, latter feature shows that the contribution of temperature to the kinetic energy of the Cooper pairs is larger for the $L=1$ state, therefore less energy is needed to heat up the system. Notice from Fig. 2 that $C_{p}\left[H=0.28 H_{c}(T)\right]<C_{p}\left[H=0.27 H_{c}(T)\right]$ since $\Delta J\left[H=0.30 H_{c}(T)\right]>\Delta J\left[H=0.27 H_{c}(T)\right]$. 


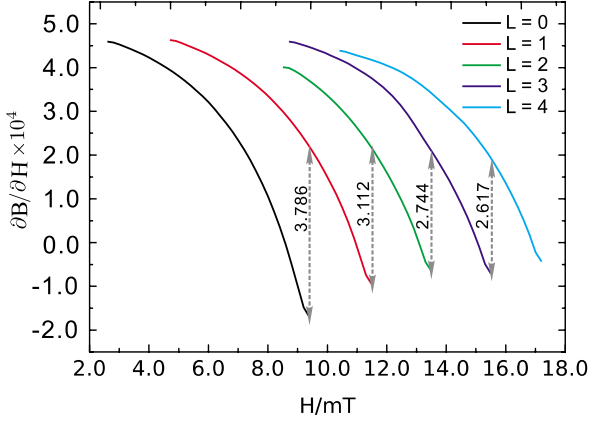

FIG. 6. (Color online) The magnetic susceptibility $\left(\partial B_{i} / \partial H\right)_{T}$ as a function of the applied magnetic field at temperature $T=0.5 T_{c}$, for different vortex states. The $L \rightarrow L+1$ transitions are shown in the figure, and the corresponding values of the jump of the magnetic susceptibility between two subsequent phases are given.

The jumps in the heat capacity between the different vortex states can be expressed more precisely using thermodynamic arguments. The theory of the specific heat in the mixed state is already well established, for the case of extreme type-II superconductors where screening is negligible (see Ref. 15). The discontinuity in the specific heat at the given phase transition can then be calculated as

$$
C_{i}-C_{j}=\frac{-T}{4 \pi}\left(\frac{d H^{*}}{d T}\right)^{2}\left[\left(\frac{\partial B_{i}}{\partial H}\right)_{T}-\left(\frac{\partial B_{j}}{\partial H}\right)_{T}\right],
$$

where $B$ stands for magnetic induction. Here we apply the above expression to the transition between the different vortex states, where $i$ represents the vortex state of vorticity $L$ just below the field $H_{L+1}^{u p}$, and $j$ represents the $L+1$ vortex state at the field just above $H_{L+1}^{u p}$. Clearly, $\left(\partial B_{i} / \partial H\right)_{T}=1+4 \pi\left(\partial M_{i} / \partial H\right)_{T}$. Therefore, we conclude that we will be able to predict the size of the jump of the specific heat taken that we know $H^{*}(T)=H_{L+1}^{u p}$ and the magnetization of each vortex state, and vice versa. From the calorimetry calculation of the specific heat we can derive the magnetic susceptibility $\left(\partial B_{i} / \partial H\right)_{T}{ }^{34}$ In Fig. 6 we show the magnetic susceptibility for each vortex state as a function of the applied magnetic field, corresponding to the diagrams of Fig. 2. We calculated $\left(\partial B_{i} / \partial H\right)_{T}$ using Eq. (5) and compared the jump in susceptibility with the result from Eq. (6). We found discrepancy of just $10 \%$, although the sample is mesoscopic and type I, none of which is included in the original theory from Ref. 15.

In the second part of our calculation, we focus on the influence of a constant perpendicular magnetic field on the heat capacity at the superconducting/normal transition (critical temperature, height, width of the transition, etc.). We fix the applied magnetic field and then calculate the specific heat while scanning temperature. For a better comparison with existing experimental data, we apply two strategies. In one, we sweep down the temperature starting from the normal state under given magnetic field which corresponds to the experimental field-cooled (FC) regime. In another, we start from zero temperature and sweep it up under fixed magnetic field [zero-field-cooled (ZFC) procedure]. The two cases are physically very different, as shown in Fig. 7(a). In the FC
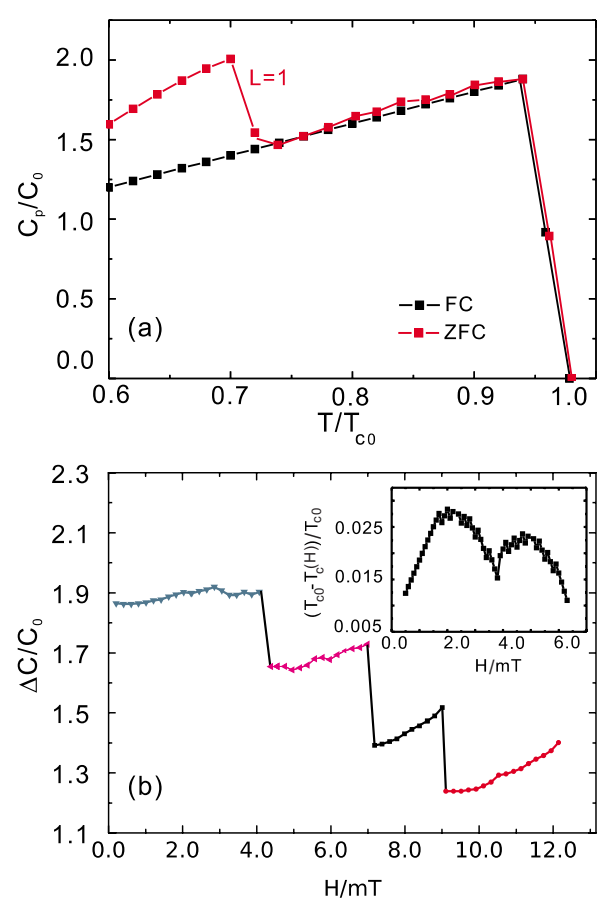

FIG. 7. (Color online)(a) $C_{p}(T)$ plots under fixed field $H=0.014 H_{c}(0)$ in field-cooled and zero-field-cooled regimes, for the sample with the same parameters as in Fig. 2. (b) $\Delta C(H)$ at the $\mathrm{S} / \mathrm{N}$ phase transition as a function of the applied magnetic field (different vortex states are shown in different colors). Inset shows the oscillation of the critical temperature as a function of applied magnetic field, derived from the observed discontinuity of $C_{p}(T)$.

regime, when the system is cooled down from the normal state, it remains in the first nucleated $L$ state as $T$ is swept down (in the present case, $L=0$ ). Therefore, we observed no features in the heat capacity, except at the $\mathrm{S} / \mathrm{N}$ phase transition. In the ZFC regime, when increasing temperature, the vorticity of the system may change. Namely, in the shown case, we found the $L=1$ state at low temperatures, which becomes unstable at temperature $T=0.68 T_{c 0}$ and a transition to $L=0$ is observed through the jump in $C_{p}(T)$.

We also considered the dependence of the jump in $C_{p}(T)$ $(\Delta C)$ at the $\mathrm{S} / \mathrm{N}$ transition on the applied magnetic field. In bulk samples, $\Delta C$ shows a monotonous decrease with $H,{ }^{5}$ while in mesoscopic superconducting systems this is not the case. We calculated $C_{p}(T)$ for a series of magnetic fields $H$, ranging from 0 to $0.40 \mathrm{H}_{c 2}$, in increasing temperature, and recorded the value of $\Delta C$ at each $T=T_{c}(H)$. As shown in Fig. 7(b), the found discontinuity is largest for the Meissner state, and decreases in a steplike manner for the states with higher vorticity. Simultaneously, we record the critical temperature vs. applied field [shown in the inset of Fig. 7(b)]. This temperature is defined as the temperature at which the discontinuity of the heat capacity takes place. ${ }^{35}$ The critical temperature $T_{c}(H)$ exhibits a cusplike, but decreasing, behavior (usually called Little-Parks oscillations, although those were found in transport measurements $\left.{ }^{30}\right)$, the $\Delta C(H)$ exhibits a discontinuity at each cusp of $T_{c}(H)$, i.e., for each new vortex state. The stability region for each $L$-state is approximately $0.1 H_{c 2}$, which is in good agreement with the experimental results of Ref. 14, adding the magnetic flux through the disk 
of about $\Phi_{0}$. Note that corresponding theoretical work was done in Ref. 12, where the modulated $\Delta C(H)$ was calculated for disks but no discontinuities were found. This difference most likely can be attributed to the different temperature dependence taken in the Gibbs free energy, but may also result from a full consideration of the nonlinear terms and the inclusion of the 3D demagnetization in our approach, instead of the 2D linearized simulation without screening effects taken in Ref. 12. We find that before each discontinuity in increasing field, the value of $\Delta C_{p}$ increases. This can be understood from the fact that the difference of magnetic susceptibility between the normal state and the different vortex states drops with increase of the vorticity, while for fixed vortex state this difference increases with applied magnetic field.

\section{HEAT CAPACITY OF MESOSCOPIC SUPERCONDUCTING RINGS}

In this section, we apply our numerical approach to study the behavior of the heat capacity of superconducting rings, both in the regimes of constant temperature and of constant magnetic field. The geometric parameters of the studied rings are: the outer and inner diameters are $D=1100 \mathrm{~nm}$ and $D_{0}=748 \mathrm{~nm}$, respectively (thus of width $w=176 \mathrm{~nm}$ ), and thickness $d=30 \mathrm{~nm}$. For the coherence length $\xi(0)$ and penetration depth $\lambda(0)$, we take 182 and $70 \mathrm{~nm}$, respectively. ${ }^{10}$ Being thin, these superconducting rings can be considered as a two-dimensional system, as all relevant quantities are distributed homogeneously across the sample in the $z$-direction. To accommodate this in the theoretical formalism, we use the effective Ginzburg-Landau parameter $\kappa_{e}=\kappa^{2} \xi / d$ in Eq. (3). The unit of the heat capacity in the calculations remains $C_{0}$, and in the present case equals $1.92 \mathrm{pJ} / \mathrm{K}$ (for an array of $N=2.47 \times 10^{6}$ rings, see Ref. 10 ).

First, we perform calculations for the heat capacity when varying the magnetic field, in the range from 0 to $6 \mathrm{mT}$, at fixed temperature $T=0.8 T_{c 0}$. The energy of the vortex state in a ring decreases and then increases with magnetic field due to the switching of the current from Meissner to vortex like at the energy minimum. Here, the heat capacity $C_{p}(H)$ shows corresponding oscillatory behavior, as shown in Fig. 8. In the considered field range, the system was stabilized in states $L=0, L=1$ and $L=2$, in increasing magnetic field. The found period of oscillations is c.a. $3 \mathrm{mT}$, which is in conformity with the oscillation period of $\Delta H=3.17 \mathrm{mT}$ measured in the experiment of Bourgeois et al. ${ }^{10}$ In our theoretical simulations, the observed period of $H$ corresponds to a flux of one flux quantum $\Phi_{0}$ through the circular area of diameter $D_{\text {eff }}=937 \mathrm{~nm},{ }^{26,31}$ thus larger than the inner diameter of the rings. Note that in Fig. 8 the superconducting heat capacity is minimal in the absence of magnetic field-it ascends for increasing applied magnetic field, contrary to the experimental observation. Latter discrepancy between our calculation and the experiment comes from the theoretical definition of the heat capacity $C_{p}$ which relates only to the superconducting condensate while $C_{\text {total }}$ was used in the experiment.

The magnetic field dependence of the critical temperature $T_{c}$ of the $\mathrm{S} / \mathrm{N}$ transition is a known signature of the Little-

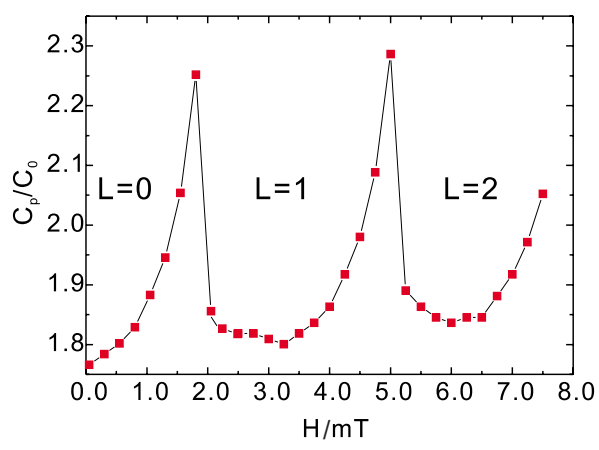

FIG. 8. (Color online) The superconducting part of the heat capacity $C_{p}(H)$ of the sample at temperature $T=0.80 T_{c 0}$ as a function of the magnetic field $H$ perpendicular to the plane of the rings. For the magnetic field in the range 0 to $6 \mathrm{mT}$, we plot the heat capacity for the $L=0,1$, and 2 states.

Parks effect in mesoscopic superconducting rings, ${ }^{5}$ which are usually studied in transport measurements. In Fig. 9 we show that the jump $\Delta C(H)$ in the heat capacity at the $\mathrm{S} / \mathrm{N}$ transition is yet another "fingerprint" of the same effect. The inset of Fig. 9 shows the $\mathrm{S} / \mathrm{N}$ phase boundary $T_{c}(H)$ that we obtain by performing scans similar to those shown in Fig. 7, for the magnetic field in the range 0 to $12 \mathrm{mT}$ (and corresponding flux of 0 to $6 \Phi_{0}$ ). The obtained period of oscillations matches roughly an added flux quantum through the ring, and the decaying behavior of the critical temperature $T_{c}(H)$ (shown in the inset of Fig. 9) agrees well with both the experimental data from Ref. 14 and the theoretical work from Ref. 20. Obtained $\Delta C(H)$ oscillations exhibit decreasing amplitude with $H$, and their period is in agreement with experiment.

Based on the understanding of the specific-heat behavior of the superconducting disk in the previous section, we now take rings of two different widths, to discuss the two different contributions to the heat capacity. The outer diameters of the rings are $D=1100 \mathrm{~nm}$, thickness $d=30 \mathrm{~nm}$, while the inner diameters are $D_{0}=600 \mathrm{~nm}$ and $400 \mathrm{~nm}$ respectively. In Figs. 10 and 11 , the heat capacity $C_{p}$ as well as its two components $C_{\Psi}$ and $C_{J}$ are plotted as function of the applied magnetic field, (where $H_{c 2}(0)=11.9 \mathrm{mT}$ ). One significant difference of the heat capacity of the ring sample and the disk ones is the different curvature of the $C_{\Psi}$ curve. From Figs. 3 and 4, one can see those curves bending up with field,

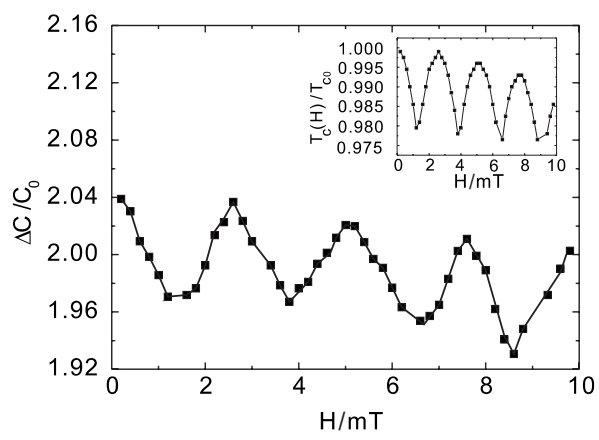

FIG. 9. The heat-capacity jump at the S/N boundary as a function of the applied magnetic field. The inset shows typical LittleParks oscillations of the critical temperature $T_{c}(H)$. 


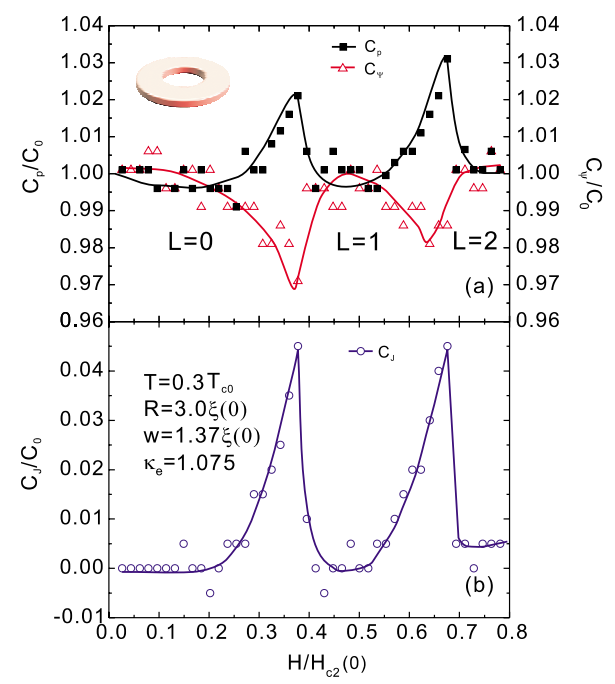

FIG. 10. (Color online) The superconducting part of the heat capacity $C_{p}(H)$, the contribution from the order parameter $C_{\Psi}(H)$ (a) and from the superconducting current $C_{J}(H)(\mathrm{b})$, of the ring with width $w=250 \mathrm{~nm}$, at temperature $T=0.30 T_{c 0}$, as a function of the magnetic field $H$. We plot the heat capacity for the $L=0,1,2$ states.

especially for higher vorticity states, and only the low vorticity states such as $L=0$ and $L=1$ are an exception where $C_{\Psi}(H)$ is flat. Here, the $C_{\Psi}$ curves bend down in the case of rings. The reason for this behavior is the penetration of the magnetic field entirely through the sample. Namely, there is no region inside the ring, where the Cooper-pair density is kept unvaried during the increase of the applied magnetic field and such region exists in the center of the disk when in the Meissner state. However, once vortices enter inside the sample, the distribution of the Cooper-pair density becomes similar in the disk and ring samples, and the tendency of bending down becomes weaker [see Fig. 10(a)], and can even flatten (Fig. 11).

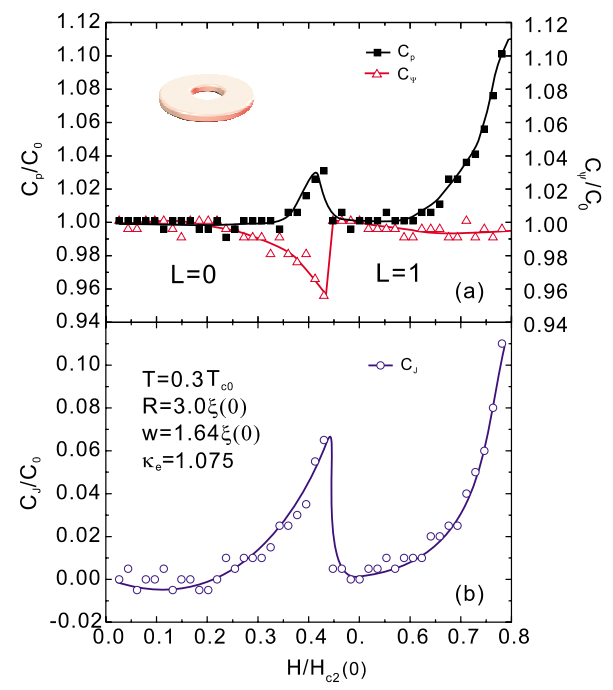

FIG. 11. (Color online) The same as Fig. 10, but now for a ring with width $w=350 \mathrm{~nm}$, and for the $L=0,1$ states. (a) The superconducting part of the heat capacity $C_{p}(H)$ and the contribution from the order-parameter variation $C_{\Psi}(H)$. (b) The kinetic contribution to heat capacity $C_{J}(H)$.
The second difference between the heat capacity of disks and loops is found for, e.g., the $L=1$ vortex state. Due to the narrow width of the ring, the magnetic field must be screened in order to prevent penetration into the sample from the inner surface, which results in Meissner currents that are maximal at the inner edge-opposite to the case of a disk, where the maximum of screening currents is always found at the outer edge. With increasing magnetic field, upon penetration of field inside the superconducting material, the current changes sign and the maximum of the current shifts to the outer edge of the ring, as is the case in disks. Thus the amplitude of the superconducting current exhibits two (displaced) maxima as a function of magnetic field, instead of just one in the case of the superconducting disk. Since heat capacity directly depends on the current profile and its temperature dependence, the specific heat as a function of magnetic field first decreases and then increases, instead of the monotonous increase found for the disk geometry. Comparing Figs. 10 and 11 , one can also find different portion of $C_{J}$ contributing to the total heat capacity, $0.04 C_{0}$ of $L=0$ and $L=1$ for $w=250 \mathrm{~nm}$ sample and $0.1 C_{0}$ of $L=1$ for $w=350 \mathrm{~nm}$ sample. Smaller $w$ also results in a smaller period of $C_{p}(H)$ oscillations due to easier fluxion quantization in a larger hole. The behavior found for $w=350 \mathrm{~nm}$ is already "disklike," where the $C_{J}$ from the $L=1$ state is much higher than that of the $L=0$ state.

\section{HEAT CAPACITY OF A MESOSCOPIC SUPERCONDUCTING CYLINDER}

Based on the understanding of the heat capacity in twodimensional systems, we can conclude that the heat capacity is strongly dependent on the vortex configuration, supercurrent distribution and even the geometry of the sample. Therefore, we explore the potential application of calorimetry to find out the "intrinsic" properties of the superconducting sample. This becomes even more important in the case of three-dimensional superconducting systems, where calorimetry measurements can give an direct insight into the vortex distribution, ${ }^{32}$ without destroying the sample or the superconducting state itself, and for arbitrary direction of applied magnetic field. To illustrate this, we apply our numerical "experiment" to a 3D mesoscopic cylinder.

The sample is a cylinder with radius $R=8 \xi(0)$, height $h=16 \xi(0)$, and the Ginzburg-Landau parameter $\kappa=0.7$. We studied the behavior of the heat capacity in the cases where the magnetic field is applied in three different directions: (1) $\theta=0^{\circ}$, along the axial direction; (2) $\theta=45^{\circ}$, along the diagonal direction and (3) $\theta=90^{\circ}$, parallel to the basal surface. Magnetization curves are calculated using an imaginary Hall bar placed above the sample, at a distance $0.5 \xi(0)$ and with size $16 \times 16 \xi(0)^{2}$. In Fig. 12 , the calculated heat capacity and the magnetization curves are plotted for case (1), as a function of the applied magnetic field. The heat capacity shows several jumps, which indicate different vortex states inside the sample just as in the $2 \mathrm{D}$ case. The magnetization curves show corresponding discontinuities, as expected.

In the case of tilted magnetic field $\left(\theta=45^{\circ}\right)$, the heat capacity shows a profoundly different behavior from what we 


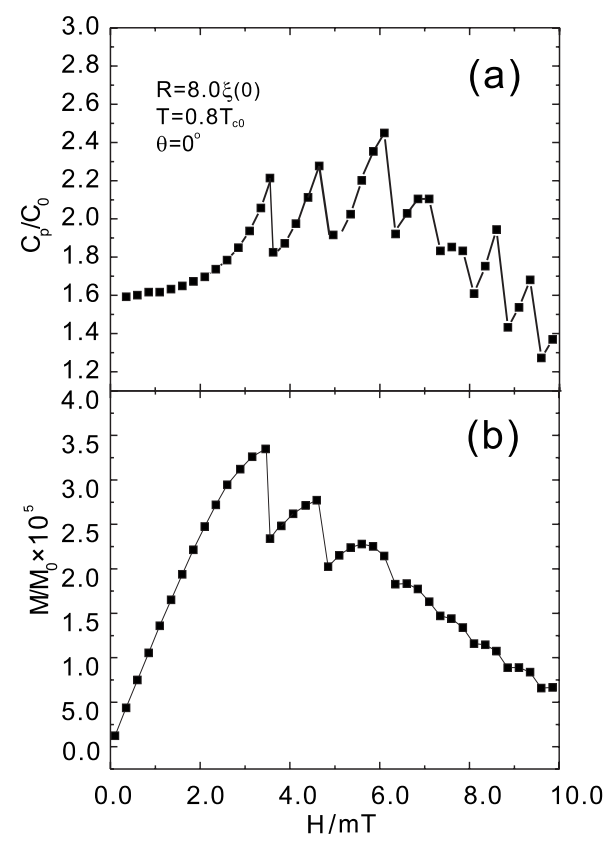

FIG. 12. The superconducting contribution to the heat capacity $C_{p}(H)$ of the sample at temperature $T=0.80 T_{c 0}$ as a function of the magnetic field $H$ in the axial direction $\left(\theta=0^{\circ}\right)$ of the threedimensional cylinder (a). The corresponding magnetization of the sample is shown as a function of $H(\mathrm{~b})$.

observed in the previous sections. Because of the coincident direction of applied field with the diagonal of the cylinder, it is energetically unfavorable for the states of odd vorticity to form inside the sample. Therefore, in the process of sweeping up the applied magnetic field, the states of $L=1$ (Fig. 13, point A), $L=3$ (Fig. 13, point C) and $L=5$ have very short stability regions. What is reflected on the heat capacity is that the states of those particular vortices are of "low" thermal stability, thus, the heat capacity of these states is much higher than that of the energetically favorable states, with even number of vortices distributed symmetrically parallel to the diagonal of the cylinder (see Fig. 13). Note also the behavior of the heat capacity of the $L=2$ state. Due to the weak stability of the $L=1$ state, the $L=2$ state appears at relatively

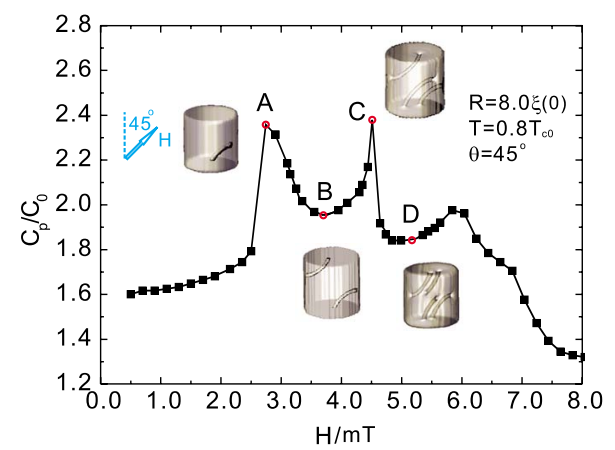

FIG. 13. (Color online) The heat capacity as a function of the applied field $H$, where the field is applied under an angle $\theta=45^{\circ}$ with respect to the axis of the cylinder. Insets show the 3D isoplots of the Cooper-pair density $\left(|\psi|^{2}=0.05\right)$, for different vortex states, corresponding to the $H$ values indicated by open circles.

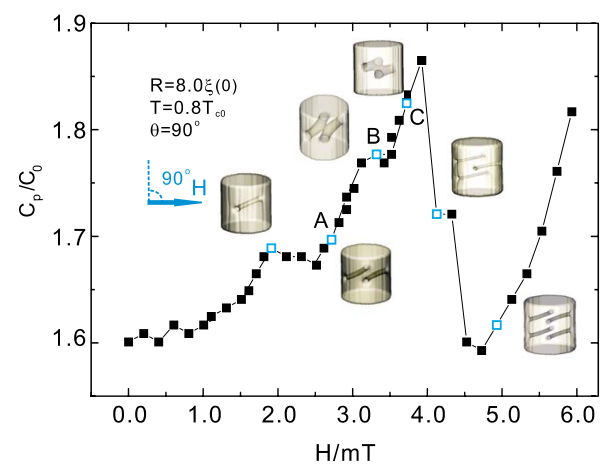

FIG. 14. (Color online) The heat capacity is plotted as a function of the applied field $\mathrm{H}$ from $H=0$ to $6.0 \mathrm{mT}$, where the field is applied with an angle $\theta=90^{\circ}$ (indicated by the arrow). Insets shows the 3D isoplots of the Cooper-pair density of the surface with $|\psi|^{2}$ $=0.05$, only for vortex states $L=1,2,3$, and 4 , which are indicated by blue empty dots.

low field, with two vortex branches located at both corners away from the diagonal line of the sample, which results in a large heat capacity. With increase of the field, both vortices are pushed closer to the diagonal center line, where the system reaches the lowest energy (point B in Fig. 13). As the field keeps increasing, vortices keep coming closer to each other, in which case, the repulsion between them becomes stronger and the heat capacity increases again. A similar process is observed also for the $L=4$ vortex state, which is indicated as point D in Fig. 13.

Finally, we discuss the case of $\theta=90^{\circ}$, where the field is applied parallel to the upper surface of the sample. The 3D isoplots of the Cooper-pair density in Fig. 14 show different vortex states for $L=1-4$. First, one can notice the short stability range of the $L=3$ state, which is caused by the symmetry breaking between the sample and the vortex distribution. Second, during the increase of the magnetic field, the heat-capacity curve of the $L=2$ state shows a short plateau. The vortex configurations are shown before, at, and after this plateau area, as points A-C, respectively in Fig. 14. One can see that before the plateau, two vortices align in plane parallel to the applied magnetic field and the upper surface. With the increase of the magnetic field, these two vortices merge together in the center (which is due to the strong interactions with the boundary at this part), and afterwards the plane of these two vortices becomes tilted at a small angle with respect to the upper surface of the sample. The small plateau in the specific-heat curve exactly records these changes of the vortex distribution.

Note therefore that the heat-capacity measurement can give a very informative description of the vortex distribution inside the 3D sample, while at the same time, the magnetization curve can be "blind" to all those processes, due to the incompatibility of the direction of the stray magnetic field and the placement of the Hall bar.

\section{CONCLUSIONS}

We developed a numerical approach to calculate the heat capacity within the Ginzburg-Landau formalism for an arbi- 
trary shape of a mesoscopic superconductor. In the present work we focused on disks and rings motivated by recent calorimetric experiments. We studied the heat-capacity dependence on magnetic field and temperature, and we proved that different flux (vortex) distribution inside the sample will produce pronounced features in the measured specific heat, different from what is known for bulk superconductors. The heat capacity exhibits large discontinuities at each phase transition, with phases being either different vortex states, or the superconducting and normal phase, in type-I as well as type-II samples. We quantitatively matched the jumps in heat capacity to changes in magnetic susceptibility, following the conjecture of de Gennes. In summary, we show that the thermodynamic properties of a mesoscopic superconductor can be manipulated by the geometry of the system, but also that the $\mathrm{S} / \mathrm{N}$ phase boundary and other phase transitions can be monitored by calorimetry. Our method is generally applicable to samples of arbitrary 3D geometry, and for magnetic field applied in any direction. We demonstrated this in the case off a mesoscopic cylinder, and show how subtle 3D changes of the vortex structure leave distinct signatures in the heat capacity. Finally, we also show the use of calorimetry in cases when conventional magnetometry is "blind."

\section{ACKNOWLEDGMENTS}

We are grateful to O. Bourgeois for useful discussions. This work was supported by the Flemish Science Foundation (FWO-Vl), the Interuniversity Attraction Poles (IAP) Program-Belgian State-Belgian Science Policy, ESF-JSPS NES program and the ESF-AQDJJ network. *francois.peeters@ua.ac.be

${ }^{1}$ V. V. Moshchalkov, L. Gielen, C. Strunk, R. Jonckheere, X. Qiu, C. V. Haesendonck, and Y. Bruynseraede, Nature (London) 373, 319 (1995).

${ }^{2}$ A. K. Geim, S. V. Dubonos, I. V. Grigorieva, K. S. Novoselov, F. M. Peeters, and V. A. Schweigert, Nature (London) 407, 55 (2000).

${ }^{3}$ D. E. Prober, Nat. Nanotechnol. 3, 459 (2008).

${ }^{4}$ D. S. Akerib et al., Phys. Rev. D 72, 052009 (2005).

${ }^{5}$ M. Tinkham, Phys. Rev. 129, 2413 (1963).

${ }^{6}$ T. McConville and B. Serin, Phys. Rev. Lett. 13, 365 (1964).

${ }^{7}$ P. Zoller and J. R. Killinger, Phys. Rev. 172, 390 (1968).

${ }^{8}$ H. Choi, K. Yawata, T. M. Haard, J. P. Davis, G. Gervais, N. Mulders, P. Sharma, J. A. Sauls, and W. P. Halperin, Phys. Rev. Lett. 93, 145301 (2004).

${ }^{9}$ T. McConville and B. Serin, Phys. Rev. 140, A1169 (1965).

${ }^{10}$ F. R. Ong, O. Bourgeois, S. E. Skipetrov, and J. Chaussy, Phys. Rev. B 74, 140503(R) (2006).

${ }^{11}$ O. Bourgeois, S. E. Skipetrov, F. Ong, and J. Chaussy, Phys. Rev. Lett. 94, 057007 (2005).

${ }^{12}$ P. S. Deo, J. P. Pekola, and M. Manninen, Europhys. Lett. 50, 649 (2000).

${ }^{13}$ W. C. Fon, K. C. Schwab, J. M. Worlock, and M. L. Roukes, Nano Lett. 5, 1968 (2005).

${ }^{14}$ F. Ong and O. Bourgeois, EPL 79, 67003 (2007).

${ }^{15}$ P. G. de Gennes, Superconductivity of Metals and Alloys (Addison-Wesley, New York, 1994).

${ }^{16}$ A. L. Fetter and P. C. Hohenberg, Superconductivity (Marcel Dekker, New York, 1969).

${ }^{17}$ K. Watanabe, T. Kita, and M. Arai, Phys. Rev. B 71, 144515 (2005).

${ }^{18}$ A. J. Bray, Phys. Rev. B 9, 4752 (1974).

${ }^{19}$ H. J. Fink and V. Grünfeld, Phys. Rev. B 23, 1469 (1981).
${ }^{20}$ X. Zhang and J. C. Price, Phys. Rev. B 55, 3128 (1997).

${ }^{21}$ V. A. Schweigert, F. M. Peeters, and P. S. Deo, Phys. Rev. Lett. 81, 2783 (1998).

${ }^{22}$ R. Kato, Y. Enomoto, and S. Maekawa, Phys. Rev. B 47, 8016 (1993).

${ }^{23}$ V. A. Schweigert and F. M. Peeters, Phys. Rev. B 57, 13817 (1998).

${ }^{24}$ D. S. Golubović, W. V. Pogosov, M. Morelle, and V. V. Moshchalkov, Appl. Phys. Lett. 83, 1593 (2003).

${ }^{25}$ D. S. Golubović, W. V. Pogosov, M. Morelle, and V. V. Moshchalkov, Europhys. Lett. 65, 546 (2004).

${ }^{26}$ B. J. Baelus, F. M. Peeters, and V. A. Schweigert, Phys. Rev. B 61, 9734 (2000).

${ }^{27}$ A. K. Geim, I. V. Grigorieva, S. V. Dubonos, J. G. S. Lok, J. C. Maan, A. E. Filippov, and F. M. Peeters, Nature (London) 390, 259 (1997).

${ }^{28}$ C. P. Bean and J. D. Livingston, Phys. Rev. Lett. 12, 14 (1964).

${ }^{29}$ J. Provost, E. Paumier, and A. Fortini, J. Phys. F: Met. Phys. 4, 439 (1974).

${ }^{30}$ R. D. Parks and W. A. Little, Phys. Rev. 133, A97 (1964).

${ }^{31}$ S. V. Yampolskii, F. M. Peeters, B. J. Baelus, and H. J. Fink, Phys. Rev. B 64, 052504 (2001).

${ }^{32}$ A. R. de C. Romaguera, M. M. Doria, and F. M. Peeters, Phys. Rev. B 75, 184525 (2007).

${ }^{33}$ To change $\kappa$ in our simulations, we fix the coherence length and vary the penetration depth.

${ }^{34}$ Note however that $\left(\partial B_{i} / \partial H\right)_{T}$ diverges at the phase-transition point.

${ }^{35}$ The bulklike linear dependence of the transition temperature on the critical field $H_{c 2}, T_{c, b} \approx\left[1-H_{c 2}(T) / H_{c 2}(0)\right] T_{c 0}$ is subtracted from the actual result, in order to highlight the modulation of the critical temperature by the applied magnetic field. 